\title{
A large deletion in RPGR causes XLPRA in Weimaraner dogs
}

\author{
Regina Kropatsch ${ }^{1 *}$, Denis A. Akkad ${ }^{1}$, Matthias Frank ${ }^{2}$, Carsten Rosenhagen ${ }^{3}$, Janine Altmüller ${ }^{4,5}$, Peter Nürnberg ${ }^{4,6,7}$, \\ Jörg T. Epplen ${ }^{1,8}$ and Gabriele Dekomien ${ }^{1}$
}

\begin{abstract}
Background: Progressive retinal atrophy (PRA) belongs to a group of inherited retinal disorders associated with gradual vision impairment due to degeneration of retinal photoreceptors in various dog breeds. PRA is highly heterogeneous, with autosomal dominant, recessive or X-linked modes of inheritance. In this study we used exome sequencing to investigate the molecular genetic basis of a new type of PRA, which occurred spontaneously in a litter of German short-hair Weimaraner dogs.

Results: Whole exome sequencing in two PRA-affected Weimaraner dogs identified a large deletion comprising the first four exons of the X-linked retinitis pigmentosa GTPase regulator (RPGR) gene known to be involved in human retinitis pigmentosa and canine PRA. Screening of 16 individuals in the corresponding pedigree of short-hair Weimaraners by $\mathrm{qPCR}$, verified the deletion in hemizygous or heterozygous state in one male and six female dogs, respectively. The mutation was absent in 88 additional unrelated Weimaraners. The deletion was not detectable in the parents of one older female which transmitted the mutation to her offspring, indicating that the RPGR deletion represents a de novo mutation concerning only recent generations of the Weimaraner breed in Germany.
\end{abstract}

Conclusion: Our results demonstrate the value of an existing DNA biobank combined with exome sequencing to identify the underlying genetic cause of a spontaneously occurring inherited disease. Identification of the genetic cause has allowed the development of a diagnostic test, which should help to eradicate the PRA causing mutation from the respective canine line. Thus, planning of future pairings is facilitated and manifestation of this type of PRA can be prevented.

Keywords: Progressive retinal atrophy, Exome sequencing, RPGR (retinitis pigmentosa GTPase regulator) gene, Weimaraner

\section{Plain English Summary}

Progressive retinal atrophy (PRA) affects cats and dogs, initially causing night blindness, followed by gradual visual loss and finally blindness. PRA comprises a large group of genetically heterogeneous retinal diseases with similar clinical appearance but differing in age of onset and rate of disease progression. Mutations in more than 20 genes are known to cause canine PRA, but the genetic cause of PRA in several affected breeds still remains to be identified. Most forms of canine PRA are inherited in an autosomal recessive manner, caused by mutations in the same gene transmitted from both parents. Autosomal dominant

\footnotetext{
* Correspondence: regina.kropatsch@rub.de

${ }^{1}$ Department of Human Genetics, Ruhr-University, Universitätsstraße 150,

44801 Bochum, Germany

Full list of author information is available at the end of the article
}

inheritance, in which one mutation suffices to cause the disease, is rarely observed. Three X-linked forms have been found to be caused by different mutations in the canine retinitis pigmentosa GTPase regulator (RPGR) gene.

Since a new type of PRA was diagnosed in a single litter of Weimaraner dogs in March 2015, we investigated all genes of two affected males in comparison with healthy controls. We analysed the DNA of all coding parts of the genes (known as exons) using exome sequencing analysis. A large gap was identified in the RPGR gene, i.e. a deletion comprising the first four exons. The X-linked $R P G R$ gene is known to be involved in both human retinitis pigmentosa and canine PRA. Screening of affected and healthy Weimaraners revealed the new mutation in hemizygous state in one affected male and in heterozygous state in six mildly affected or asymptomatic female dogs, whereas it 
was absent in all unrelated Weimaraners investigated. The deletion was not present in the parents of one older female which transmitted the mutation to her offspring. This indicates that the RPGR gene was newly mutated in this female or in the parental sperm or egg, respectively. The established DNA test allows easy mutation detection and helps the breeder community to plan future pairings for the next generations of Weimaraners without this type of PRA.

\section{Background}

Retinal dystrophies are a common cause of blindness in both human beings and purebred dogs (Canis familiaris). The most typical form of canine dystrophy is PRA which is an equivalent in phenotype and disease progression to retinitis pigmentosa (RP) in man [1]. PRA encompasses a large group of genetically heterogeneous retinal dystrophies that share a similar phenotype of progressive visual impairment, finally leading to blindness. Initially, rod photoreceptor vision is affected resulting in night blindness, followed by progressive loss of cone photoreceptors with deterioration in daytime vision [2]. In other forms of retinal diseases such as cone-rod dystrophy and achromatopsia, daytime vision is impaired prior to night vision [3]. PRA has been documented in more than 100 different dog breeds [2]. To date, mutations in at least 20 genes have been associated with PRA [4]. However, the genetic cause of PRA in several affected breeds still remains to be identified. Most forms of canine PRA are inherited in an autosomal recessive manner, but an autosomal dominant trait [5] as well as three X-linked forms are also known [6-8] which arose independently as single mutation events [9]. The different forms of PRA can be classified by the age of onset of night blindness and the rate of disease progression (i.e., vision loss) [2].

In Germany, in March 2015, several litter mates of Weimaraners (Fédération Cynologique Internationale group 7, section 1, standard No. 99) aged $\sim 2.5$ years were ophthalmologically diagnosed with a bilateral retinal dystrophy resembling PRA. The Weimaraner, originally designated as Weimar pointer, represents a native old German breed of hunting dogs. Its origin can be traced back to the 18th century when the first breed standard was established [10]. With about 500 puppies per year in Germany (http://www.vdh.de/ueber-den-vdh/ welpenstatistik/; Verband für das Deutsche Hundewesen, VDH) the Weimaraner does not have a particularly small population base. Hence, the molecular genetic basis of PRA in this breed was studied using next generation sequencing (NGS). A large deletion was identified in the RPGR gene, mutations of which have been reported to cause X-linked RP (XLRP) in man [11] and Xlinked PRA in dogs [7].

\section{Methods \\ Dogs}

All investigated dogs originated from the common breeding population of pure-bred short-hair Weimaraners. Veterinarians who were specialized and experienced in ophthalmological diseases (Dortmunder Kreis, DOK) confirmed the PRA status of affected male dogs $(n=3$; Fig. 1b) and of female dogs with mild symptoms $(n=3)$ by ophthalmoscopy, as documented in certificates of eye examinations. For all other dogs, general veterinarian examinations revealed normal sight as prerequisite for breeding. In total, blood samples of 108 dogs were received from the owners either submitted to the Weimaraner DNA biobank (established at the Department of Human Genetics, Ruhr-University Bochum, Germany) or sent to our institute for other research projects such as investigation of genetic variability in the Weimaraner population. Written informed consent was obtained from the owners for sample collection and genetic investigations. Sample collection was performed by practicing veterinarians according to international guidelines for the use of laboratory animals. As the DNA stems from the blood of client-owned dogs that underwent routine veterinary examinations including venipuncture, animal experiments were not

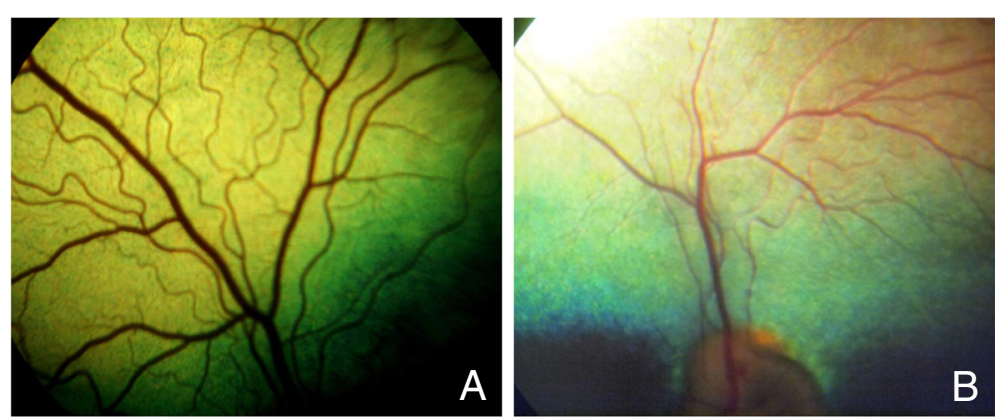

Fig. 1 Ophthalmoscopic appearance of the central fundus. a Healthy unrelated female Weimaraner dog ( 3 years) with a normally appearing fundus (right eye). b PRA-affected male Weimaraner dog ( 2.5 years) showing the typical landmarks of PRA with vessel attenuation primarily of the arterioles in the papillary area and a hyperreflective tapetum lucidum (left eye) 
performed and, therefore,approval by an ethical committee was not necessary. Genomic DNA was isolated from peripheral blood cells using a standard protocol [12]. To test matrilineal inheritance of PRA in Weimaraners, mitochondrial genome sequencing was performed in one PRA-affected dog and his mother.

For whole exome sequencing, we used the genomic DNA of four male Weimaraners, two of them diagnosed with PRA and two healthy control dogs older than the early age of onset ( $\sim 2.5$ years) of this PRA form in Weimaraner dogs. To verify co-segregation, identified variants were investigated in both PRA-affected dogs and their parents by Sanger sequencing. For extended segregation analysis, a pedigree was available comprising 18 Weimaraners, including the two male dogs diagnosed with PRA, their parents, maternal grandparents and two siblings as well as three further breeding partners of the mother and seven half-siblings (Fig. 2a). A cohort of 88 healthy control Weimaraners (41 male, 47 female) which were unrelated to the PRA-affected line for at least five generations was used for validation purposes in case of co-segregation of putative disease causing variants and structural abnormalities using restriction fragment length polymorphism (RFLP) and quantitative real-time PCR (qPCR) analyses, respectively. $90 \%$ of these 88 control dogs were born at least 10 years ago, the youngest male control dogs are 8 and 9 years old by now, respectively. With an average life expectancy of about 12 years, most of the controls are already deceased today. None of the male (and female) control dogs had been reported to the DNA biobank registry as showing restrictions in the visual system to date. All potentially inherited medical impairments are registered in the DNA biobank files of the respective dogs.

\section{NGS-based mutation screening}

Whole exome sequencing was performed on four Weimaraner DNAs (including two of the dogs diagnosed with PRA). The canine exome library consisted of a custom track ordered from Agilent (Santa Clara, CA, USA) as a $54 \mathrm{Mb}$ design. Therefore, tracks of the UC Santa Cruz Genomics Institute (UCSC) Genome Browser from the dog (Canis familiaris) whole genome shotgun (WGS) assembly v.2.0 (CanFam2 May 2005) were used as references for the Ensembl Genome Browser (http:// www.ensembl.org/index.html) as well as tracks of the National Center for Biotechnology Information (NCBI) reference sequence database (https://www.ncbi.nlm.nih.gov/refseq/rsg/), human protein alignments and spliced expressed sequence tags (EST) that lie outside the tracks of the Ensembl Genome Browser. Sequencing was performed on an Ilumina HiSeq 2000 platform (Illumina Inc., San Diego, CA) at the Cologne Center for Genomics (CCG, Köln, Germany) using a single lane per sample, generating paired-end reads of 2x100 nucleotides (nt) in length and yielding an average coverage of $\sim 60 \mathrm{x}$. Fastq files were further processed using the Next$\mathrm{GENe}^{\circ}$ software (Softgenetics, State College, PA, USA) according to the manufacturer's protocol. Sequences were aligned to the available reference tracks CanFam2 and xenoRefGene.txt corresponding to the best blast alignment of known genes from other species (available from UCSC Genome Browser, http://hgdownload.cse.ucsc.edu/ goldenPath/canFam2/database/) using standard settings. $99.1 \%$ of the reads matched to the reference genome with $82.9 \%$ matching perfectly.

Mutations were called using the NextGENe ${ }^{\circ}$ software standard settings for a retinal candidate gene panel as well as for the entire exome. For initial retinal candidate gene analysis, a panel of all known RP-associated and retina-specific genes was created based on retinal candidate genes listed by Kastner et al. [13] and complemented by candidate genes from the Retinal Information Network (RetNet, http://www.sph.uth.tmc.edu/retnet/) database as well as from recent literature. Since the mode of inheritance of PRA in the Weimaraner breed was not obvious initially, retinal candidate genes were included with different modes of inheritance (autosomal dominant, autosomal recessive and X-linked). Candidate variants were identified by variant comparison and copy number variation (CNV) tools integrated in the $\mathrm{NextGENe}^{\bullet}$ software. For variant comparison, PRAaffected individuals were compared to unaffected individuals assuming recessive and dominant modes of inheritance-including the possibility of a heterozygous carrier status in the unaffected dogs in case of recessive inheritance. For CNV analyses, PRA-affected individuals were compared to unaffected individuals using the multiple controls option and the best match mode.

Variations or structural aberrations were considered as candidates for further analyses if they were present at least in heterozygous state in both affected individuals but not in the unaffected ones (dominant model) or if they were present in homozygous state in the PRA-affected dogs and absent or in heterozygous state in the unaffected ones (recessive model). Potential mutations were evaluated in more detail by visualization of the bam-files (binary version of sequence alignment map) with the integrative genomics viewer (IGV; [14]). Diverse online tools and genome browsers, including UCSC Genome Browser (http://genome.cse.ucsc.edu/), Ensembl, Online Mendelian Inheritance in Man (OMIM, http://www.ncbi.nlm.nih.gov/omim), RetNet and GeneCards (http://www.genecards.org/) were also used to provide details about conservation, gene expression, gene function and relevant pathogenic information in man. 


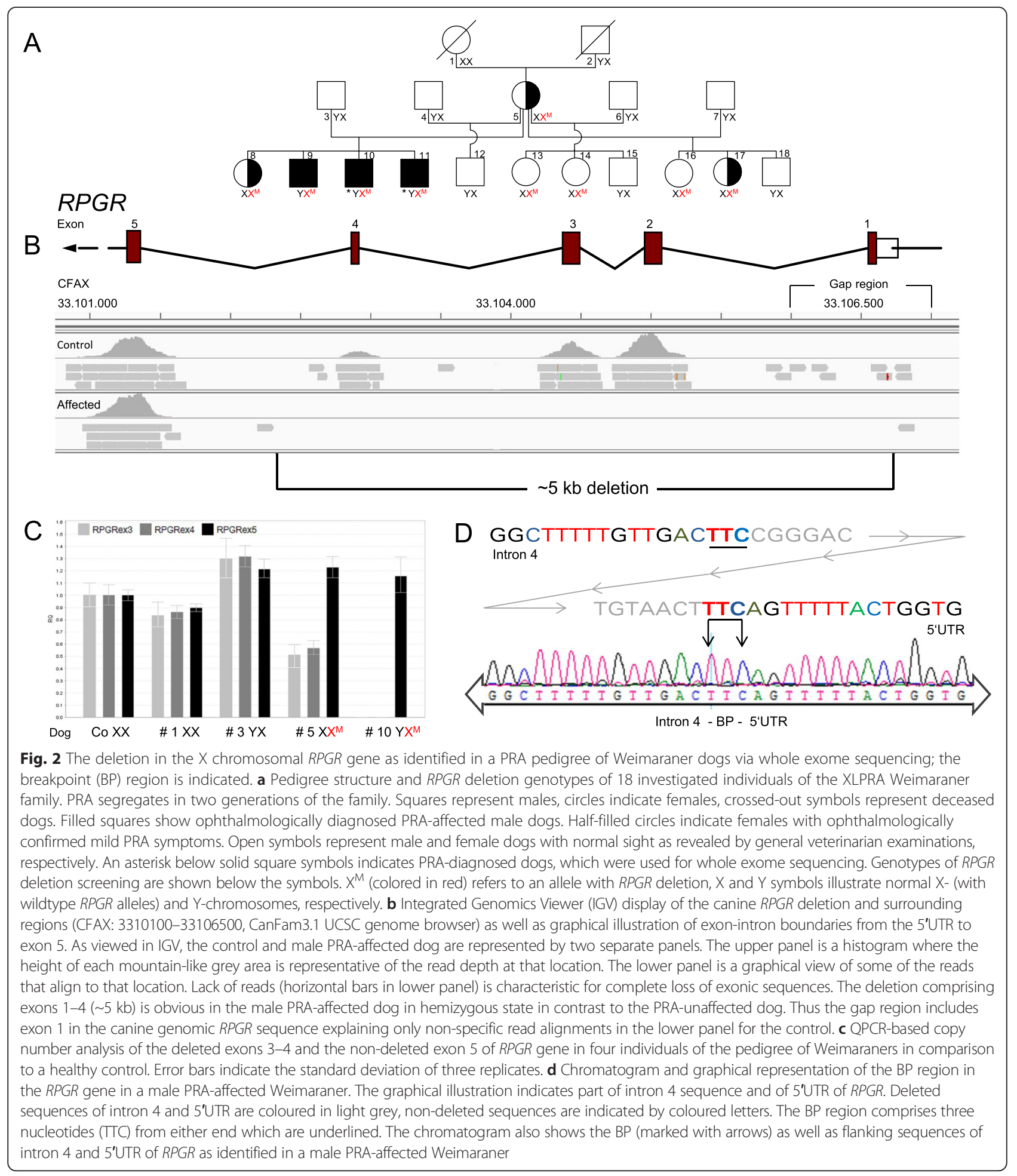

\section{Validation of candidate variants and structural abnormalities}

Sanger DNA sequencing of the entire mitochondrial genome (mtDNA) and potential candidate single nt variants was carried out using the Big Dye Terminator kit v3.1 on an ABI 3500XL Genetic Analyzer (Applied Biosystems,
Darmstadt, Germany), as previously described [15]. Sequences were evaluated with the Variant Reporter software (Applied Biosystems) and the SeqManII software (DNAstar, Madison, WI, USA). For further validation of potential candidate variants, RFLP analyses were performed as previously described [15]. For verification of structural 
abnormalities, qPCR was run on the StepOnePlus real time-PCR system (Applied Biosystems) using the KAPA SYBR $^{\circ}$ FAST ABI Prism ${ }^{\text {tw }}$ One-Step qRT-PCR reagent (peqLab, Erlangen, Germany) as described by the manufacturer. Baseline and threshold values were set automatically and cycle threshold $(\mathrm{CT})$ values were determined using the StepOnePlus software (Applied Biosystems). Gene copy numbers were calculated using the $\Delta \Delta C T$ method [16] with normalization to the X-chromosomal dystrophin $(D M D)$ gene. Primer sequences used for $\mathrm{qPCR}$ are listed in the Additional file 1.

Primers used for candidate gene and mtDNA sequencing, RFLP, qPCR analyses and breakpoint PCR were designed with the LightScanner Primer Design Software v1.0 (Idaho Technology Inc., Salt Lake City, Utah, USA) according to the CanFam2 genome sequence with minimization of possible single nucleotide polymorphisms (SNPs) at the primer binding sites.

Parentage testing was performed using the microsatellite marker based parentage testing kit Canine Genotypes Panel 2.1 on the ABI 3500XL Genetic Analyzer (Applied Biosystems) according to the manufacturer's protocol. For allele sizing, the GeneScan ${ }^{\text {Tw }} 600$ LIZ $^{\circ}$ Size Standard v2.0 (Thermo Fisher Scientific, Dreieich, Germany) was used. Raw data of microsatellite marker testing were automatically analysed by the ABI 3500XL Genetic Analyzer (Applied Biosystems) and evaluated using the GeneMapper v4.1 software (Applied Biosystems). To confirm the results of the parentage testing kit Canine Genotypes Panel 2.1, DNA profiling was also performed with parentage testing kit Canine Genotypes Panel 1.1 (Thermo Fisher Scientific) which is licensed for parentage testing by the International Society for Animal Genetics.

\section{$R P G R$ genomic sequence and mutation analyses}

The canine genomic RPGR sequences (chrX: 3305637133105037, CanFam3.1) were compared to an in silicopredicted RPGR mRNA in humans (UCSC genome browser accession NM_000328.2) in order to verify exon-intron boundaries. Since the genomic database sequence includes a gap comprising the 5 'UTR, exon 1 with the start codon and parts of intron 1 in the canine $R P G R$ gene, the respective region was amplified by longrange PCR (expand high fidelity PCR system; Roche, Mannheim, Germany) and sequenced with the Sanger method. The newly identified sequences of the RPGR gap region were verified by comparisons with the NCBI database using the Basic Local Alignment Search Tool (BLAST, http://blast.ncbi.nlm.nih.gov/Blast.cgi).

For deletion size estimation in RPGR, the newly identified 5' untranslated region (5'UTR) and part of intron 4 (IVS4) were amplified by PCR using different primer combinations and sequenced with the Sanger method in order to identify the recombination breakpoints. One primer pair was used for breakpoint PCR (diagnostic test) in order to verify qPCR-based deletion screening results. Primer sequences used for recombination breakpoint analysis are listed in the Additional file 1.

\section{Results}

\section{Phenotype of PRA in Weimaraner dogs}

Three male German Weimaraner litter mates were suspected to be affected by PRA upon ophthalmoscopic examination. They showed a significant progressive night blindness with slightly unsafe behaviour in twilight because of visual disturbances. Other than in healthy Weimaraners (Fig. 1a), ophthalmological investigations revealed PRA-like abnormalities such as diffuse hyperreflectivity in the tapetum ludidum, depigmentation in the non-tapetal part of the fundus and generalised vascular attenuation (Fig. 1b). All three PRA-affected males were $\sim 2.5$ years of age at the time of diagnosis.

Three female Weimaraner dogs (including the mother of the PRA-affected litter mates, one sibling and one half-sibling) showed mild symptoms of PRA at ophthalmological investigations such as patchy and diffuse areas of hypo- and hyperreflectivity in the dorsal tapetum ludidum, whereas retinal vessels and non-tapetal fundus were not altered (data not shown). No other symptoms of PRA were found in the female sibling and the mother aged $\sim 2.5$ years and $\sim 8$ years at diagnosis, respectively. However, the mother was only investigated after the PRA diagnosis in her offspring. For the female halfsibling, $\sim 5$ years of age at diagnosis, the owners had noticed slightest changes in visual capability. Progression of retinal degeneration was not documented within the limited time span since PRA was initially diagnosed in the Weimaraner (March to December 2015).

\section{Candidate gene and mtDNA screening}

Due to the unknown inheritance mode of the PRA form in the Weimaraner breed and in order to test the hypothesis of non-mendelian inheritance, the mitochondrial genome (16,727 nt) was analysed for one PRA-affected Weimaraner and his mother. This analysis revealed identical mtDNA haplotypes (the matrilineage) in 100 homoplasmic and eight heteroplasmic variations in both individuals and no differences to healthy dogs in the NCBI database. Thus, no mutation associated with PRA was obvious (data not shown).

\section{Next generation and Sanger sequencing}

Whole exome sequencing was performed in two male PRA-affected and two male healthy control Weimaraner dogs. In total, 351,538 sequence variants were identified. Initial retinal candidate gene panel filtering for 346 genes (see Additional file 1) causing autosomal dominant, recessive or X-linked inherited retinal diseases 
revealed no PRA causing mutation for the PRA-affected dogs. Therefore, comparison analyses of all 351,538 whole exome sequence variants of PRA-affected versus control dogs assuming recessive or dominant inheritance were performed by using the comparison tool of the Next$\mathrm{GENe}^{\circ}$ software. In total, 6,805 potentially recessive and 15,135 potentially dominant variants were identified. Most variants were excluded as causative for PRA due to information concerning poorly conserved locations, nonretina-specific gene expression and function from diverse public databases. However, four potential variants (two nonsense and two missense mutations) in the genes endoplasmic reticulum aminopeptidase 2 (ERAP2), DnaJ/ Hsp40 homolog, subfamily c, member 4 (DNAJC4), exportin 4 (XPO4) and dihydropyrimidine dehydrogenase $(D P Y D)$ were not excluded by the aforementioned criteria and subsequently verified by Sanger sequencing. Analyses of these four sequence variants demonstrated segregation in both PRA-affected dogs and their parents. However, RFLP analyses of a cohort of 88 unrelated control Weimaraners excluded all four variants as causative for PRA since they were identified in numerous healthy individuals. Next, comparisons of whole exome CNVs of PRA-affected versus control Weimaraners were performed by using the $\mathrm{CNV}$ tool of the NextGENe ${ }^{\circ}$ software. In total, 341 deletions and 552 duplications were identified and verified with the IGV viewer. Among these, a large deletion of exon 1-4 of the X-linked RPGR gene was identified in the PRA-affected Weimaraners, which was not found in the controls (Fig. 2b). RPGR is a PRA candidate gene (RetNet database) since mutations in this gene have been described to cause both XLRP in man [11] and X-linked PRA in dogs [7].

\section{Mutation screening}

The presence of the RPGR deletion was verified in both male PRA-affected dogs (by qPCR analysis of the deleted exons 3-4 and the non-deleted exon 5) in comparison to their parents (\#3, \#5), their maternal grandmother (\#1) and a healthy control. Compared to the control with normal copy number for all three exons, the PRAaffected $\operatorname{dog}(\# 10)$ lacked these exons, and his mother (\#5) showed just one copy for exons 3 and 4, while two copies for exon 5 were identified in both of them. For all three exons, two copies were evidenced in his grandmother (\#1) and one copy in his father (\#3; Fig. 2c). QPCR-based screening of the deleted exon 4 in 13 further individuals of the pedigree of the affected dogs revealed no copy for another male sibling (\#9) and one copy for another female sibling (\#8) as well as four female half-siblings (\#13-14, \#16-17). Two copies of exon 4 were shown for the maternal grandfather (\#2), three male half-siblings (\#12, \#15,\#18) and the three breeding partners of the mother (\#4, \#6-7). In total, qPCR analyses verified the deletion in the three affected males and the three mildly affected females in the investigated Weimaraner pedigree (Fig. 2a). Additionally, the deletion was demonstrated in three female dogs which so far have not revealed any clinical symptoms. In 88 unrelated control Weimaraners, the presence of exon 4 was proven by normal copy numbers (data not shown). After identification of RPGR deletion breakpoints, a breakpoint PCR (diagnostic test) was performed for all 18 individuals of the pedigree confirming the results of $R P G R$ deletion screening as identified by $\mathrm{qPCR}$ and indicating that the deletion comprises exons $1-4$ of the RPGR gene (data not shown).

In order to verify that the X-linked PRA in the investigated Weimaraner pedigree is caused by a de novo mutation, we investigated the biological parentage of both the mother and the maternal grandparents of the PRAaffected dogs by using the microsatellite marker based parentage testing kits Canine Genotypes Panel 2.1 and Canine Genotypes Panel 1.1. No inconsistencies concerning the ancestry were identified in the investigated individuals.

\section{Mutation detection}

In order to determine the genomic size of the RPGR deletion, the recombination breakpoints in IVS4 and $5^{\prime}$ UTR were analysed by PCR amplification employing various primer sets. However, via UCSC database comparisons, a gap region was identified in the canine $R P G R$ sequence comprising 5'UTR, exon 1 with the start codon and parts of intron 1 . The sequence of this gap was analysed by long-range PCR and subsequent primer walking. The newly identified DNA sequence of this gap region comprised $846 \mathrm{nt}$ including a known long interspersed nuclear element (LINE, repeat L1_Canis1_family L1, UCSC genome browser) which occurs several times in the canine genome (NCBI GenBank accession no KU234669). A part of the newly identified genomic sequence of exon 1 was also detected in an mRNA isoform of the RPGR gene in Canis lupus familiaris (accession NM_001003126.1). As indicated by sequencing, the breakpoint regions comprised three nucleotides (TTC, Fig. 2d). The exact breakpoints are localized in the newly identified 5' sequence of RPGR gene (chrX: $33106747+$ 190, UCSC genome browser, CanFam3.1, September 2011 assembly) and in IVS4 (chrX: 33102324), respectively. Thus, the $R P G R$ deletion comprised a maximum size of 5,006 nt in the mutation-carrying Weimaraners.

\section{Discussion}

In the spring of 2015, ophthalmological examinations had revealed retinopathy in two male symptomatic Weimaraners from a single litter born in 2012. Female dogs of this and another maternal litter were diagnosed 
with milder impairments. The veterinarians (at DOK) named this form of retinal disease in Weimaraner retinopathy instead of PRA, because clear progression was not documentable within the limited time span. Nonetheless, since deleterious mutations in the RPGR gene cause XLPRA, we use the original designation.

Here, whole exome sequencing was applied to map the causative mutation for this early-onset form of PRA in Weimaraners. Several litter mates had been diagnosed with PRA, but the mode of inheritance was not clear initially. One cause for RP in man are matrilineally inherited mutations in the mitochondrial genome [17]. Analysis of the latter revealed no obvious pathogenic differences in Weimaraners.

Using NGS-based whole exome analysis, a large deletion comprising the first four exons including the start codon was identified in the X-chromosomal RPGR gene in PRA-affected Weimaraners. This gene encodes a binding protein with a $\mathrm{N}$-terminal domain homologous to the regulator of chromosome condensation 1 ( $R C C 1$ ), a guanine nucleotide exchange factor for the small GTPase Ran [18], which is important for the association with binding partners [19]. RPGR transcripts undergo extensive alternative splicing e.g. in man, mouse and dog $[20,21]$ producing constitutive and open reading frame 15 (ORF15) variants with an additional exon ORF15 by using alternative polyadenylation and splice sites [22] The constitutive variant is expressed in a wide variety of tissues [20], whereas the ORF15 variant is predominantly found in the retina [22]. The retinal subcellular localization of RPGR protein is shown to be relatively consistent across several mammalian species with high enrichment in the photoreceptor connecting cilium [23]. Although the exact function of RPGR is not fully understood, studies suggest that it plays an important role in photoreceptor survival and its development [7]. RPGR has been shown to interact with a variety of ciliary proteins such as RPGRIP1 [24]. In this context six candidate genes were discussed as potential modifiers in canine XLPRA [25]. Mutations in the RPGR gene account for over $70 \%$ of X-linked RP [22, 26] and 15-20\% of all RP [27]. Exon ORF15 is known to be a mutational hotspot for XLRP, accounting for about $70 \%$ of all diseasecausing mutations in the RPGR gene in humans [28]. Two canine animal models of ORF15 frame-shift mutations have been published which resemble the human phenotype in disease onset and progression [7, 21]. Thus, the Weimaraner breed is the third naturally occurring pure-bred animal model for XLRP in addition to Siberian husky and Samoyed [7, 21]. However, it is the first one with a causative mutation localized in the highly conserved $5^{\prime}$ terminus of the RPGR gene in contrast to the mutational hotspot in exon ORF15. Since the identified RPGR deletion was not detectable in the maternal grandparents of the affected male Weimaraners, biological parentage had to be confirmed by microsatellite marker testing. The deletion was also absent in 88 unrelated Weimaraner dogs, the deletion has occurred de novo.

Based on comparisons with the location of $\mathrm{CpG}$ islands in the human RPGR reference sequence (UCSC genome browser, hg38, December 2013 assembly), we suggest that the canine RPGR promotor region is situated in the newly identified DNA sequence in the $5^{\prime}$ sequence of the gene, outside of the 5 'terminal breakpoint region. Therefore, the deletion comprised the first four exons including the start codon of the RPGR gene, suggesting that the translation initiation is impaired resulting in the lack of RPGR gene products. Previous analyses of mice lacking Rpgr showed that photoreceptor morphology was initially normal but photoreceptor cell degeneration was noted over time, suggesting that the Rpgr gene is required for long-term maintenance of photoreceptors [29]. This correlates with the PRA form studied here in Weimaraners which presented with initial PRA symptoms at $\sim 2.5$ years of age. In addition, it has been shown that a 2 nt- frameshift microdeletion causes photoreceptor degeneration in canine X-linked PRA [30]. Finally, UCSC Genome Browser comparisons with human RPGR transcripts demonstrate that a functional transcript lacking the first four exons has not been observed so far.

Our molecular genetic results of the RPGR deletion as evidenced in the Weimaraner pedigree studied (Fig. 2a) were entirely consistent with the respective results of ophthalmological investigations by DOK veterinarians. The deletion was identified in hemizygous state in three male Weimaraner litter mates all of which were ophthalmologically diagnosed with PRA and showed diffuse tapetal hyperreflectivity, attenuated vessels (Fig. 1b) and visual disturbances in twilight compared to a dog with normal central fundus (Fig 1a) and without visual impairments. In six female individuals of the pedigree the deletion was found in heterozygous state, and ophthalmological investigations in three of them revealed mild symptoms of peripheral hyperreflexia (data not shown). These findings are in accordance with a recessive mode of X-linked inheritance in which hemizygous males are usually severely affected, while female individuals carrying the deletion in heterozygous state may be asymptomatic or, in case of non-random or skewed X-inactivation, may present a mild phenotype [31].

\section{Conclusions}

The findings of the present study demonstrate the value of an existing DNA biobank combined with modern tools available for genomic mutation analyses to aid rapid identification of the genetic cause of a spontaneously occurring 
disease in a dog breed. Without the DNA biobank for Weimaraner dogs, it would have been impossible to include the deceased maternal grandparents in this study. This implies that the RPGR deletion screening would have had to be performed in a much larger population of Weimaraners. In short, a diagnostic test has been established, that allows for mutation screening for both very young and (still) healthy dogs. Thus, precise conclusions may be drawn, and the PRA mutation may be eradicated from the respective Weimaraner line.

\section{Additional file}

Additional file 1: A Candidate genes for PRA exome screening $(n=346)$.

B and $\mathbf{C}$ Primers used for breakpoint analysis (B) and $\mathrm{GPCR}(\mathbf{C})$ with their corresponding product size. (XLSX $19 \mathrm{~kb})$

\section{Acknowledgements}

The authors thank the veterinarians of the Dortmunder Kreis (DOK) for the ophthalmologic investigation of the Weimaraners. We are grateful to Michaela Hagedorn for excellent technical assistance, Dr. V. Wagner (Biofocus; Recklinghausen, Germany) for confirming pedigree data as well as Dr. S. Hoffjan for editing the entire manuscript and improving the English.

\section{Funding}

Publication costs were covered by the Open Access Publication Funds of the Ruhr-University Bochum. Apart from this we did not have any other funding sources.

\section{Availability of data and materials}

The data sets (four Fasta files) supporting the results of this article are available in the European Nucleotide Archive (ENA) repository [study accession number: PRJEB14490; http://www.ebi.ac.uk/ena/data/view/ PRJEB14490].

\section{Authors' contributions}

RK and GD carried out the molecular genetic studies, analysed the data and drafted the manuscript. JA and PN performed NGS-based exome sequencing. DAA helped to analyse the NGS data. MF and CR provided illustration of ophthalmological examinations. JTE conceived of the study, designed and managed the project and helped to draft the manuscript. All authors read and approved the final version of the manuscript.

\section{Competing interests}

The authors declare that they have no competing interests.

\section{Author details}

'Department of Human Genetics, Ruhr-University, Universitätsstraße 150, 44801 Bochum, Germany. ${ }^{2}$ Kleintierklinik Frank, Mooswaldallee 10 i, 79108 Freiburg, Germany. ${ }^{3}$ Tierärztliche Überweisungspraxis, Lortzingstraße 12, 28209 Bremen, Germany. ${ }^{4}$ Cologne Center for Genomics, University of Cologne, Weyertal 115 b, 50931 Cologne, Germany. ${ }^{5}$ Institute of Human Genetics, University of Cologne, Kerpener Str. 34, 50931 Cologne, Germany. ${ }^{6}$ Center for Molecular Medicine Cologne (CMMC), University of Cologne, Robert-Koch Str. 21, 50931 Cologne, Germany. ${ }^{7}$ Cologne Excellence Cluster on Cellular Stress Responses in Aging-Associated Diseases (CECAD), University of Cologne, Joseph-Stelzmann-Str. 26, 50931 Cologne, Germany. ${ }^{8}$ Faculty of Health, University Witten-Herdecke, Alfred-Herrhausen-Strasse 50, 58448 Witten, Germany.

Received: 23 March 2016 Accepted: 21 June 2016 Published online: 08 July 2016

\section{References}

1. Petersen-Jones S. Advances in the molecular understanding of canine retinal diseases. J Small Anim Pract. 2005;46:371-80.
2. Miyadera K, Acland GM, Aguirre GD. Genetic and phenotypic variations of inherited retinal diseases in dogs: the power of within- and across-breed studies. Mamm Genome. 2012;23:40-61.

3. Petersen-Jones SM, Komaromy AM. Dog models for blinding inherited retinal dystrophies. Hum Gene Ther Clin Dev. 2015;26:15-26.

4. Wiik AC, Ropstad EO, Ekesten B, Karlstam L, Wade CM, Lingaas F. Progressive retinal atrophy in Shetland sheepdog is associated with a mutation in the CNGA1 gene. Anim Genet. 2015;46:515-21.

5. Kijas JW, Cideciyan AV, Aleman TS, Pianta MJ, Pearce-kelling SE, Miller BJ, et al. Naturally occurring rhodopsin mutation in the dog causes retinal dysfunction and degeneration mimicking human dominant retinitis pigmentosa. Proc Natl Acad Sci U S A. 2002;99:6328-33.

6. Vilboux T, Chaudieu G, Jeannin P, Delattre D, Hedan B, Bourgain C, et al. Progressive retinal atrophy in the Border Collie: a new XLPRA. BMC Vet Res. 2008:4:10.

7. Zhang Q, Acland GM, Wu WX, Johnson JL, Pearce-Kelling S, Tulloch B, et al. Different RPGR exon ORF15 mutations in Canids provide insights into photoreceptor cell degeneration. Hum Mol Genet. 2002;11:993-1003.

8. Acland GM, Blanton SH, Hershfield B, Aguirre GD. XLPRA: a canine retinal degeneration inherited as an X-linked trait. Am J Med Genet. 1994;52:27-33.

9. Zangerl B, Johnson JL, Acland GM, Aguirre GD. Independent origin and restricted distribution of RPGR deletions causing XLPRA. J Hered. 2007;98:526-30.

10. Schmidt H. Der Jagdgebrauchshund. 1st ed. 1989

11. Shu X, Black GC, Rice JM, Hart-Holden N, Jones A, O'Grady A, et al. RPGR mutation analysis and disease: an update. Hum Mutat. 2007;28:322-8.

12. Miller SA, Dykes DD, Polesky HF. A simple salting out procedure for extracting DNA from human nucleated cells. Nucleic Acids Res. 1988;16:1215.

13. Kastner S, Thiemann I-J, Dekomien G, Petrasch-Parwez E, Schreiber S, Akkad DA, et al. Exome sequencing reveals AGBL5 as novel candidate gene and additional variants for retinitis pigmentosa in five Turkish families. Investig Ophthalmol Vis Sci. 2015:56:8045-53.

14. Thorvaldsdóttir H, Robinson JT, Mesirov JP. Integrative Genomics Viewer (IGV): high-performance genomics data visualization and exploration. Brief Bioinform. 2013;14:178-92.

15. Kropatsch R, Streitberger K, Schulte-Middelmann T, Dekomien G, Epplen JT. On ancestors of dog breeds with focus on Weimaraner hunting dogs. J Anim Breed Genet. 2011:128:64-72.

16. Livak KJ, Schmittgen TD. Analysis of relative gene expression data using real-time quantitative PCR and the 2(-Delta Delta C(T)) Method. Methods. 2001;25:402-8.

17. Phelan JK, Bok D. A brief review of retinitis pigmentosa and the identified retinitis pigmentosa genes. Mol Vis. 2000;6:116-24.

18. Roepman R, van Duijnhoven G, Rosenberg T, Pinckers AJLG, BleekerWagemakers $L M$, Bergen AAB, et al. Positional cloning of the gene for $X$-linked retinitis pigmentosa 3: homology with the guanine-nucleotideexchange factor RCC1. Hum Mol Genet. 1996;5:1035-41.

19. Neidhardt J, Glaus E, Barthelmes D, Zeitz C, Fleischhauer J, Berger W. Identification and characterization of a novel RPGR isoform in human retina. Hum Mutat. 2007;28:797-807.

20. Kirschner R, Rosenberg T, Schultz-Heienbrok R, Lenzner S, Feil S, Roepman R, et al. RPGR transcription studies in mouse and human tissues reveal a retina-specific isoform that is disrupted in a patient with X-linked retinitis pigmentosa. Hum Mol Genet. 1999;8:1571-8.

21. Zeiss CJ, Ray K, Acland GM, Aguirre GD. Mapping of X-linked progressive retinal atrophy (XLPRA), the canine homolog of retinitis pigmentosa 3 (RP3). Hum Mol Genet. 2000;9:531-7.

22. Vervoort R, Lennon A, Bird AC, Tulloch B, Axton R, Miano MG, et al. Mutational hot spot within a new RPGR exon in X-linked retinitis pigmentosa. Nat Genet. 2000;25:462-6.

23. Hong D-H, Pawlyk B, Sokolov M, Strissel KJ, Yang J, Tulloch B, et al. RPGR isoforms in photoreceptor connecting cilia and the transitional zone of motile cilia. Invest Ophthalmol Vis Sci. 2003;44:2413-21.

24. Megaw RD, Soares DC, Wright AF. RPGR: Its role in photoreceptor physiology, human disease, and future therapies. Exp Eye Res. 2015;138:1-10.

25. Guyon R, Pearce-Kelling SE, Zeiss CJ, Acland GM, Aguirre GD. Analysis of six candidate genes as potential modifiers of disease expression in canine XLPRA1, a model for human X-linked retinitis pigmentosa 3. Mol Vis. 2007;13:1094-105

26. Bader I, Brandau O, Achatz H, Apfelstedt-Sylla E, Hergersberg M, Lorenz B, et al. X-linked retinitis pigmentosa: RPGR mutations in most families with 
definite $X$ linkage and clustering of mutations in a short sequence stretch of exon ORF15. Investig Ophthalmol Vis Sci. 2003;44:1458-63.

27. Breuer DK, Yashar BM, Filippova E, Hiriyanna S, Lyons RH, Mears AJ, et al. A comprehensive mutation analysis of RP2 and RPGR in a North American cohort of families with X-linked retinitis pigmentosa. Am J Hum Genet. 2002;70:1545-54

28. Sharon D, Sandberg MA Rabe WW, Stillberger M, Dryja TP, Berson EL. RP2 and RPGR mutations and clinical correlations in patients with X-linked retinitis pigmentosa. Am J Hum Genet. 2003;73:1131-46.

29. Hong DH, Pawlyk BS, Shang J, Sandberg MA, Berson EL, Li T. A retinitis pigmentosa GTPase regulator (RPGR)-deficient mouse model for X-linked retinitis pigmentosa (RP3). Proc Natl Acad Sci U S A. 2000;97:3649-54.

30. Beltran WA, Hammond P, Acland GM, Aguirre GD. A frameshift mutation in RPGR exon ORF15 causes photoreceptor degeneration and inner retina remodeling in a model of $X$-linked retinitis pigmentosa. Invest Ophthalmol Vis Sci. 2006;47:1669-81.

31. Brown CJ, Robinson WP. The causes and consequences of random and non-random X chromosome inactivation in humans. Clin Genet. 2000;58:353-63.

\section{Submit your next manuscript to BioMed Central} and we will help you at every step:

- We accept pre-submission inquiries

- Our selector tool helps you to find the most relevant journal

- We provide round the clock customer support

- Convenient online submission

- Thorough peer review

- Inclusion in PubMed and all major indexing services

- Maximum visibility for your research

Submit your manuscript at www.biomedcentral.com/submit 